\title{
Loss of coherence in double-slit diffraction experiments
}

\author{
A. S. Sanz ${ }^{*}$ and F. Borondo ${ }^{\dagger}$ \\ Departamento de Química, C-IX, Universidad Autónoma de Madrid, Cantoblanco-28049, Madrid, Spain \\ M. J. Bastiaans \\ Faculteit Elektrotechniek, Technische Universiteit Eindhoven, Postbus 513, 5600 MB Eindhoven, The Netherlands
}

(Received 14 October 2003; revised manuscript received 9 December 2004; published 14 April 2005)

\begin{abstract}
The effects of incoherence and decoherence in a double-slit experiment are studied using both optical and quantum-phenomenological models. The results are compared with experimental data obtained with cold neutrons.
\end{abstract}

DOI: 10.1103/PhysRevA.71.042103

PACS number(s): 03.65.Yz, 03.65.Ta, 03.75.Dg

\section{INTRODUCTION}

Coherence is an important building block in physics, common to optics and quantum mechanics. In classical optics, it simply implies a phase condition related to interference. Its lack is related to the nature of the source of light (nonmonochromaticity) and/or the geometrical shape of the objects placed on the source-detector pathway $[1,2]$. In this sense, incoherence can be regarded as a "nondynamical" effect. On the other hand, the same concept acquires a more general meaning in quantum mechanics, where it has to be reconciled with the statistical interpretation of the wave function. When two or more states are coherently superposed their properties qualitatively differ from those exhibited by the isolated components, this being a distinctive feature of the quantum behavior [3].

Due to its remarkable relevance in the modern quantum theory of information and quantum computation [4], a topic of much recent interest is the understanding of how quantum systems lose their coherence, decoherence being the most widely accepted mechanism [5]. By decoherence we understand the irreversible emergence of classical properties in a quantum system through its "dynamical" interaction with an environment or bath $[6,7]$. This idea is better understood by splitting the Hamiltonian describing the full system in three terms:

$$
\hat{H}=\hat{H}_{A}+\hat{H}_{B}+\hat{V}_{A B},
$$

where $\hat{H}_{A}$ and $\hat{H}_{B}$ are the Hamiltonians governing the evolution of system and environment, respectively, and $\hat{V}_{A B}$ accounts for the coupling between them. This last term leads to a fast cancellation of the off-diagonal elements of the system's reduced density matrix, which is studied by averaging (i.e., tracing) the full-system density matrix over the environment degrees of freedom.

\footnotetext{
*Present address: Chemical Physics Theory Group, Department of Chemistry, University of Toronto, Toronto, Canada M5S 3H6. Email address: asanz@chem.utoronto.ca

†Email address: f.borondo@uam.es

*Email address: m.j.bastiaans@tue.nl
}

In general, the visibility of an experimental diffraction pattern can be considered the result of combining incoherence and decoherence. In this paper we analyze the influence of these two mechanisms using a theoretical model applied to the double-slit experiment with cold neutrons carried out by Zeilinger et al. [8]. By means of a detailed optical study of the neutron beam [1], we conclude that incoherence and decoherence are both needed in order to explain the loss of coherence found in the experiment, this being the main result of our work. Decoherence is introduced here using a simple phenomenological theoretical model that assumes an exponential damping of the interferences [9], and that has only one empirical parameter (the coherence degree). It should be stressed out that this model is based solely on first principles (except for the estimation of the coherence parameter). Other authors have treated the effects of incoherence in similar diffraction experiments with $\mathrm{He}$ atoms [10] and $\mathrm{He}$ clusters [11] from an optical point of view, and also the effects of decoherence in fullerene diffraction [12].

The organization of the paper is as follows. To make the paper self-contained, we briefly review in Sec. II the experiment carried out by Zeilinger et al. [8]. Section III is devoted to the optical description of this experiment, and in Sec. IV we present our quantum calculations, whose results are compared with the experimental data. Finally, the main conclusions derived from this work are summarized in Sec. V.

\section{THE EXPERIMENT}

The double slit used by Zeilinger et al. [8] consisted of a highly absorbing boron wire mounted into the opening gap of a single slit. The dimensions of this arrangement (hereafter labeled $O$ ) are $a_{1}|d| a_{2}=21.9|104.1| 22.5 \mu \mathrm{m}$ (left slit|boron wire|right slit). The wavelength of the neutron beam, $\lambda_{\mathrm{dB}}=18.45 \AA$ (with a bandwidth $\Delta \lambda= \pm 1.40 \AA$ ), was selected by means of an entrance single slit $(C)$ of width $w$ $=20 \mu \mathrm{m}$, located at a distance $z=5 \mathrm{~m}$ before $O$. After crossing the double slit and a pathway $v=5 \mathrm{~m}$, neutrons are made to pass through a scanning single slit $(D)$ of width $w_{0}$ $=20 \mu \mathrm{m}$ before reaching the detector (located behind $D$ ).

The reported results are reproduced in Fig. 1. From them, a value for the fringe visibility of $\mathcal{V}_{\text {expt }}=0.583$ is obtained. This magnitude is defined $[1,2]$ as 


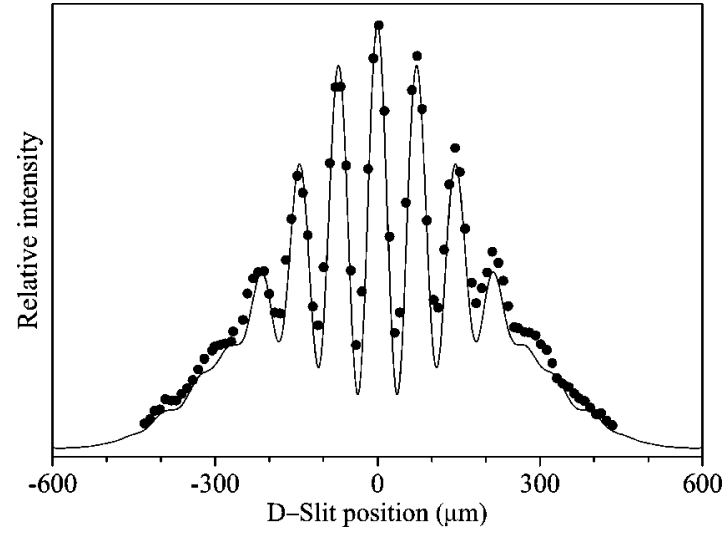

FIG. 1. Experimental results obtained by Zeilinger et al. [8] for the double-slit diffraction of cold neutrons (solid circles), and theoretical intensity patterns obtained by assuming two finite-size identical slits [see Eq. (15)] (solid line).

$$
\mathcal{V}=\frac{I_{\max }-I_{\min }}{I_{\max }+I_{\min }},
$$

$I_{\max }$ and $I_{\min }$ being the intensities corresponding to the central maximum and the first minimum next to it, respectively. This experimental lack of visibility cannot be attributed to simple causes, such as nonconvergence of the intensity pattern at $D$ or the size difference between slits. In the first case, $D$ is far enough from $O$, and the intensity pattern is well defined (it displays almost vanishing minima). In the second case, the difference between $a_{1}$ and $a_{2}$ is too small ( $\left.\simeq 3 \%\right)$, and the incoherence effects due to this asymmetry are irrelevant. In the following sections the physical factors leading to this high value for the loss of coherence in the experiment are examined.

\section{THE OPTICAL TREATMENT}

Studies on neutron interferometry can be accurately carried out by means of classical optics [13] provided that energy is low and spin effects are negligible. Since classical optics only accounts for incoherence effects, this approach is of great help in our case in order to discern whether or not decoherence effects are relevant. This analysis will also provide the physical conditions that the quantum model for the possible decoherence must satisfy. Therefore, we first analyze here the optics of the neutron beam to establish the relevance of incoherence effects in the diffraction pattern.

The coherence of light can be understood as the effect of stationary stochastic processes [14], i.e., random fluctuations in the amplitude and phase of the interfering waves of light in time. Although the source of that stochasticity is irrelevant for the optical analysis, it turns out to be crucial in the quantum theory of decoherence [5]. The correlation between two processes taking place at different points and times is given by the (mutual) coherence function [15-17], $\Gamma\left(x_{1}, x_{2} ; \tau\right)$, which is only a function of the time difference due to stationarity. From it, the power spectrum [16,17] or crossspectral density function [18]

$$
S\left(x_{1}, x_{2} ; \omega\right)=\int \Gamma\left(x_{1}, x_{2} ; \tau\right) e^{i \omega \tau} d \tau
$$

can be defined. This is the optical analog of the quantum density matrix. Like it, $S\left(x_{1}, x_{2} ; \omega\right)=S^{*}\left(x_{2}, x_{1} ; \omega\right)$ for the offdiagonal elements, while the diagonal ones $S(x, x ; \omega)$ are real and give the intensity distribution of light at a frequency $\omega$. Therefore, the power spectrum is the essential element in this optical treatment.

Since Eq. (3) refers to the neutron beam, $\omega$ can be substituted by $\lambda$, without loss of generality. As in Ref. [8], in the theoretical description of the experiment we will take into account the pass of the neutron beam through $C, O$, and $D$. If $C$ is identified with a neutron source that can be assumed as incoherent [8], i.e., the intensity at each point along the aperture does not depend on any other point, then

$$
S_{C}\left(x_{1}, x_{2} ; \lambda\right)=q\left(x_{1} ; \lambda\right) \delta\left(x_{1}-x_{2}\right) .
$$

Here, $q(x, \lambda) \geqslant 0$ is the average intensity at $x$. When no absorption takes place at the borders of $C$ (i.e., each point of the aperture produces the same intensity) and $\lambda$ is independent of the position along this aperture, $q(x ; \lambda)$ is given by

$$
q(x ; \lambda)=\frac{1}{w} s(\lambda), \quad|x| \leqslant w / 2,
$$

with $s(\lambda)$ describing the spectral profile of the neutron beam. It should be emphasized that to keep the explicit dependence on $\lambda$ is important, since the source is not totally monochromatic. As will be seen below, the spectral profile plays a key role on the shape of the measured intensity at $D$ within this optical treatment.

From $C$ to $O$ the neutron beam propagates freely according to the following point-spread function [16]:

$$
h_{C O}(x, \xi)=e^{i k(x-\xi)^{2} / 2 z},
$$

where $x$ and $\xi$ refer to the spatial coordinates at $O$ and $C$ [19], respectively, and $k=2 \pi / \lambda$. In this way, the power spectrum just before $O$ becomes

$$
\begin{aligned}
S_{O}^{\prime}\left(x_{1}, x_{2} ; \lambda\right) & =\int h_{C O}\left(x_{1}, \xi_{1}\right) h_{C O}^{*}\left(x_{2}, \xi_{2}\right) S_{C}\left(\xi_{1}, \xi_{2} ; \lambda\right) d \xi_{1} d \xi_{2} \\
& =\bar{q}(\Delta x ; \lambda) e^{i k\left(x_{1}^{2}-x_{2}^{2}\right) / 2 z}
\end{aligned}
$$

where

$$
\bar{q}(\Delta x ; \lambda)=\operatorname{sinc}\left[k\left(x_{1}-x_{2}\right) w / 2 z\right] s(\lambda),
$$

with $\Delta x=x_{1}-x_{2}$ and $\operatorname{sinc} \beta=\sin \beta / \beta$. As can be seen, the finite size of the source gives rise to a coherence in the neutron beam, which manifests as a typical single-slit diffraction pattern at $O$. The first minima of this correlation pattern [20] take place at $\Delta x= \pm z \lambda_{\mathrm{dB}} / w \simeq \pm 461 \mu \mathrm{m}$. Therefore, the spatial width illuminated by its central maximum will be relatively larger than the extension covered by the double-slit arrangement. This means that the two diffracted beams will display a slight divergence, a fact that must be included as a source of incoherence in the quantum model (see Sec. IV). 
The double slit acts as a modulator of the incoming wave with a modulation function $m(x)$. Thus, the power spectrum at $O$ can be written as

$$
S_{O}\left(x_{1}, x_{2} ; \lambda\right)=m\left(x_{1}\right) m^{*}\left(x_{2}\right) S_{O}^{\prime}\left(x_{1}, x_{2} ; \lambda\right) .
$$

After crossing the double slit the two diffracted beams described by $S_{O}$ propagate freely until reaching $D$. There, the power spectrum $S_{D}\left(x_{1}, x_{2} ; \lambda\right)$ is computed by replacing, in Eq. (7), $h_{C O}(x, \xi)$ and $S_{C}\left(\xi_{1}, \xi_{2} ; \lambda\right)$ by $h_{O D}(x, \xi)$ and $S_{O}\left(\xi_{1}, \xi_{2} ; \lambda\right)$, respectively. The diagonal elements of $S_{D}$ give the intensity distribution, $I(x ; \lambda)$, right before the neutron beam crosses $D$. For example, in the case of two identical very narrow slits (such that the modulation function can be expressed as the sum of two $\delta$ functions) separated from each other a distance $\bar{d}=d+\left(a_{1}+a_{2}\right) / 2$, one obtains

$$
\begin{aligned}
S_{D}\left(x_{1}, x_{2} ; \lambda\right)= & {[\cos (k \Delta \bar{d} / 2 v) s(\lambda)} \\
& \left.+\bar{q}(\bar{d} ; \lambda) \cos \left[k\left(x_{1}+x_{2}\right) \bar{d} / 2 v\right]\right] e^{i k\left(x_{1}^{2}-x_{2}^{2}\right) / 2 v},
\end{aligned}
$$

from which

$$
I(x ; \lambda)=[1+\operatorname{sinc}(k \bar{d} w / 2 z) \cos (k \bar{d} x / v)] s(\lambda) .
$$

The separation between two consecutive maxima that results from Eq. (11) is $v \lambda_{\mathrm{dB}} / \bar{d} \simeq 73 \mu \mathrm{m}$, which is in good agreement with the experimental value of Fig. 1. However, the value of the fringe visibility, $\mathcal{V}=0.881$, is still far from the experimental value. Moreover, the amplitude of the oscillations is constant, in strong disagreement with the experimental pattern.

If the finite size of $D$ is taken into account, the measured intensity for a position $x$ of the detector results

$$
I_{0}(x ; \lambda)=\frac{1}{w_{0}} \int_{x-w_{0} / 2}^{x+w_{0} / 2} I\left(x^{\prime} ; \lambda\right) d x^{\prime},
$$

which decreases the fringe visibility, but leaves the profile of the interference pattern unaffected. The nonmonochromaticity of the neutron beam leads to a subsequent integration over the spectral bandwidth. Taking into account the optical relation $k=\omega / c$, this integral can be carried out easily by assuming $\omega=2 \pi c / \lambda$, and substituting the corresponding quantities by $\lambda_{\mathrm{dB}}$ and $\Delta \lambda=2.80 \AA$. Thus, in the particular case of a uniform spectral profile over the bandwidth $\Delta \omega$, one obtains

$$
\begin{aligned}
I_{0}(x)= & \frac{1}{\Delta \omega} \int_{\omega-\Delta \omega / 2}^{\omega+\Delta \omega / 2} I_{0}\left(x ; \omega^{\prime}\right) d \omega^{\prime} \\
\propto & 1+\operatorname{sinc}(k \bar{d} w / 2 z) \operatorname{sinc}\left(k \bar{d} w_{0} / 2 v\right) \\
& \times \operatorname{sinc}\left[\left(\Delta \lambda / \lambda_{\mathrm{dB}}\right)(k \bar{d} x / 2 v)\right] \cos (k \bar{d} x / v),
\end{aligned}
$$

where the approximation of constant sinc functions (with $\lambda$ $=\lambda_{\mathrm{dB}}$ ) has been used, since these functions vary slowly within the integration interval (about $4 \%$ with respect to their value at $\lambda=\lambda_{\mathrm{dB}}$ ). The results obtained with this expression are much better than those for Eq. (11) but still far from the experimental data. In particular a value of $\mathcal{V}=0.772$ is ob- tained, and the averaging process introduces a certain modulation with respect to the constant-amplitude intensity of Eq. (11). However, the decay in the intensity pattern as the absolute value of the detector position increases is not satisfactory. This is a general feature [i.e., independent of the spectral profile determined by $s(\lambda)]$ coming from the assumption of point slits at $O$.

The modulation of the intensity pattern is a border effect caused by the finite size of the slits, while the oscillations are the result of the interference of two spatially separated wave fronts. This can be easily shown by considering that the slits are coherently illuminated (i.e., the modulation function is described by the sum of two hat functions), and their size is relatively small [21]. In this way, one can assume that Eq. (11) still holds, and introduces the sinc function caused by a finite-size slit into it, thus obtaining

$$
\begin{aligned}
I(x, \lambda) \propto & {\left[\operatorname{sinc}^{2}\left(k \bar{a} \eta^{-} / 2 v\right)+\operatorname{sinc}^{2}\left(k \bar{a} \eta^{+} / 2 v\right)\right.} \\
& +2 \operatorname{sinc}\left(k \bar{a} \eta^{-} / 2 v\right) \operatorname{sinc}\left(k \bar{a} \eta^{-} / 2 v\right) \\
& \times \operatorname{sinc}(k \bar{d} w / 2 z) \cos (k \bar{d} x / v)] s(\lambda) .
\end{aligned}
$$

Here, for the sake of simplicity, both slits are considered with the same width $\bar{a}=\left(a_{1}+a_{2}\right) / 2$, and $\eta^{ \pm}=x \pm v \bar{d} / 2 b$. Using the same arguments, an equivalent expression for Eq. (13) can be calculated,

$$
\begin{aligned}
I_{0}(x) \propto & \operatorname{sinc}^{2}\left(k \bar{a} \eta^{-} / 2 v\right)+\operatorname{sinc}^{2}\left(k \bar{a} \eta^{+} / 2 v\right) \\
& +2 \operatorname{sinc}\left(k \bar{a} \eta^{-} / 2 v\right) \operatorname{sinc}\left(k \bar{a} \eta^{-} / 2 v\right) \operatorname{sinc}(k \bar{d} w / 2 z) \\
& \times \operatorname{sinc}\left(k \bar{d} w_{0} / 2 v\right) \operatorname{sinc}\left[\left(\Delta \lambda / \lambda_{\mathrm{dB}}\right)(k \bar{d} x / 2 v)\right] \cos (k \bar{d} x / v) .
\end{aligned}
$$

The corresponding results are shown as the solid line in Fig. 1. As can be seen, the intensity pattern fits much better the experimental results, presenting only a small discrepancy for the central minima. However, the fringe visibility $\mathcal{V}=0.760$ still does not fully account for the value obtained experimentally, although it is improved with respect to the previous cases. Therefore, the most important conclusion derived from this phenomenological analysis of the experiment is that, although different causes contribute to the incoherence of the neutron beam, they are not sufficient to explain the experimental results. The remaining contribution should then be attributed to dynamical causes, i.e., decoherence, which will be analyzed in the next section.

One final consideration is worth mentioning. The finite size of the slits is an important factor to take into account in the formulation of the quantum model. Indeed, a general phenomenological form for the measured intensity accounting for the loss of coherence can be postulated from Eq. (15) as

$$
\mathcal{I}(x)=\mathcal{I}_{1}(x)+\mathcal{I}_{2}(x)+2 \mathcal{A} \mathcal{I}_{12}(x) \cos (k \bar{d} x / v),
$$

where $\mathcal{I}_{i}(x)$ is the intensity function produced by the slit $i$ (with $i=1,2), \mathcal{I}_{12}(x)$ the intensity function modulating the interference, $\mathcal{A}$ the coherence degree or damping coefficient, and the cos function is the term purely due to the interference between the two slits. Notice that the coherence degree and 
fringe visibility are not the same unless both partial waves contribute equally to the intensity, as happens in Eq. (11), where $\mathcal{I}_{1}(x)=\mathcal{I}_{2}(x)=\mathcal{I}_{12}(x)=1 / 2$.

\section{THE QUANTUM TREATMENT}

Optical models are attractive because of their simplicity (and accuracy when used in association with fitting procedures). However, they do not deal with the loss of coherence due to dynamical processes, such as those taking place in the regions where the neutron beam evolves freely. The effect produced by such processes is what we call decoherence. In general, decoherence is understood as a complex effect concerning the phase of a many-body wave function [22]. For example, according to the model proposed by Caldeira and Leggett [23], decoherence arises from the interaction of the system with a bath of harmonic oscillators linearly coupled to the system. The collisions between particles belonging to the system and to the environment constitute another mechanism proposed to explain the loss of coherence $[6,7]$ in physical processes. Although these scattering events may be individually quite inefficient, when taking place in large numbers they may lead to an exponential damping of the off-diagonal elements of the system reduced density matrix, since

$$
\left.\frac{\partial \widetilde{\rho}_{n m}}{\partial t}\right|_{\text {scattering }}=-\lambda \tilde{\rho}_{n m}(t)
$$

where $\lambda=\Gamma\left(1-\left\langle\psi_{0}\left|S_{m}^{\dagger} S_{n}\right| \psi_{0}\right\rangle\right), \Gamma$ is the collision rate, and the scattering process between states $|n\rangle$ and $|m\rangle$ is described by the corresponding $S$-matrix element.

According to the physical considerations made in the previous section, in our quantum-mechanical description of the process we only consider the evolution of the neutron beam from $O$ to $D$, assuming that both outgoing partial waves at $O$ are coherent (i.e., finite-size slits). Moreover, as mentioned above, these partial waves differ in width (because of the different slit sizes), and then move apart, separating along the two opposite directions (notice, however, that the component of this motion is relatively small). These initial conditions are responsible for the incoherence. Taking this into account, the evolution of the neutron beam after passing through $O$ (and without being affected by an external environment) can be described at any subsequent time [24] as

$$
\left|\Psi^{(0)}\right\rangle_{t}=c_{1}\left|\psi_{1}\right\rangle_{t}+c_{2}\left|\psi_{2}\right\rangle_{t}
$$

where $\left|\psi_{i}\right\rangle_{t}$ is the partial wave emerging from slit $i$, and $\left|c_{1}\right|^{2}+\left|c_{2}\right|^{2}=1$ at any time. In the coordinate representation, the density matrix associated with this wave function is

$$
\rho_{t}^{(0)}\left(\mathbf{r}, \mathbf{r}^{\prime}\right)=\Psi_{t}^{(0)}(\mathbf{r})\left[\Psi_{t}^{(0)}\left(\mathbf{r}^{\prime}\right)\right]^{*}
$$

with $\Psi_{t}^{(0)}(\mathbf{r})=\left\langle\mathbf{r} \mid \Psi^{(0)}\right\rangle_{t}$. The diagonal terms of Eq. (19) give the probability density or measured intensity

$$
\begin{aligned}
\rho_{t}^{(0)} & =\rho_{t}^{(0)}(\mathbf{r}, \mathbf{r}) \\
& =\left|c_{1}\right|^{2}\left|\psi_{1}\right|_{t}^{2}+\left|c_{2}\right|^{2}\left|\psi_{2}\right|_{t}^{2}+2\left|c_{1}\right|\left|c_{2}\right|\left|\psi_{1}\right|_{t}\left|\psi_{2}\right|_{t} \cos \delta_{t},
\end{aligned}
$$

where $\delta_{t}$ is the time-dependent phase difference between the two partial waves.

When the action of an environment is considered, the wave function (18) no longer describes the evolution of the full system. Since our main interest focuses on the question of how the environment affects the coherence of the system by gradually suppressing the (nonclassical) oscillatory term in Eq. (20), we will assume conditions of elastic scattering [25]. Accordingly, only the states describing the environment change during the scattering process, while the system states remain unchanged. In particular, in our case both partial waves will propagate as free Gaussian wave packets since they are Gaussians initially (see below). In this way, the initial coherent state

$$
|\Psi\rangle=\left|\Psi^{(0)}\right\rangle \otimes\left|E_{0}\right\rangle
$$

becomes an entangled state with the form

$$
|\Psi\rangle_{t}=c_{1}\left|\psi_{1}\right\rangle_{t} \otimes\left|E_{1}\right\rangle_{t}+c_{2}\left|\psi_{2}\right\rangle_{t} \otimes\left|E_{2}\right\rangle_{t}
$$

at any subsequent time. Here, $\left|\psi_{1}\right\rangle$ and $\left|\psi_{2}\right\rangle$ are coherent wave packets (or superpositions of them),

$$
G(x, z) \propto e^{-\left(x-x_{0}\right)^{2} / 4 \sigma_{x}^{2}+i p_{x} x} e^{-\left(z-z_{0}\right)^{2} / 4 \sigma_{z}^{2}+i p_{z} z},
$$

where $x(z)$ is the parallel (perpendicular) coordinate (with respect to the plane of the double slit), $x_{0}\left(z_{0}\right)$ is the center of the wave packet along the $x(z)$ axis, $p_{x}\left(p_{z}\right)$ is the parallel (perpendicular) component of the momentum, and $\sigma_{x}\left(\sigma_{z}\right)$ is the width of the wave packet along $x(z)$. In the case of quasiplane waves, the corresponding wave function can be constructed as a coherent superposition of Gaussian wave packets distributed over the width covered by each slit. On the other hand, notice from Eq. (22) that, due to the initial coherence, $\left|E_{1}\right\rangle=\left|E_{2}\right\rangle=\left|E_{0}\right\rangle$. In our case, this initial state for the environment is chosen as $\left|E_{0}\right\rangle=|\mathbb{I}\rangle$.

In order to calculate the diffraction intensity, the reduced density matrix for the system is obtained by tracing the fullsystem density matrix over the environment states,

$$
\hat{\tilde{\rho}}_{t}=\sum_{i}\left\langle E_{i}\left|\hat{\rho}_{t}\right| E_{i}\right\rangle_{t}
$$

In the coordinate representation this operation is equivalent to carrying out the integral of the total density matrix over all the $3 N$ degrees of freedom, $\left\{\mathbf{r}_{i}\right\}_{i=1}^{N}$, of the environment,

$$
\tilde{\rho}_{t}\left(\mathbf{r}, \mathbf{r}^{\prime}\right)=\int\left\langle\mathbf{r}, \mathbf{r}_{1}, \ldots \mathbf{r}_{n} \mid \Psi\right\rangle_{t}\left\langle\Psi \mid \mathbf{r}^{\prime}, \mathbf{r}_{1}, \ldots \mathbf{r}_{n}\right\rangle d \mathbf{r}_{1} \cdots d \mathbf{r}_{n}
$$

By substituting Eq. (22) into Eq. (24), one obtains then the reduced density matrix 


$$
\begin{aligned}
\tilde{\rho}_{t}\left(\mathbf{r}, \mathbf{r}^{\prime}\right)= & \left(1+\left|\alpha_{t}\right|^{2}\right)\left[\left|c_{1}\right|^{2} \psi_{1 t}(\mathbf{r}) \psi_{1 t}^{*}\left(\mathbf{r}^{\prime}\right)+\left|c_{2}\right|^{2} \psi_{2 t}(\mathbf{r}) \psi_{2 t}^{*}\left(\mathbf{r}^{\prime}\right)\right] \\
& +2 \alpha_{t} c_{1} c_{2}^{*} \psi_{1 t}(\mathbf{r}) \psi_{2 t}^{*}\left(\mathbf{r}^{\prime}\right)+2 \alpha_{t}^{*} c_{2} c_{1}^{*} \psi_{2 t}(\mathbf{r}) \psi_{1 t}^{*}\left(\mathbf{r}^{\prime}\right),
\end{aligned}
$$

where $\alpha_{t}={ }_{t}\left\langle E_{2} \mid E_{1}\right\rangle_{t}$. From Eq. (26) we obtain the measured intensity

$$
\begin{aligned}
\tilde{\rho}_{t}= & \left(1+\left|\alpha_{t}\right|^{2}\right)\left[\left|c_{1}\right|^{2}\left|\psi_{1}\right|_{t}^{2}+\left|c_{2}\right|^{2}\left|\psi_{2}\right|_{t}^{2}\right. \\
& \left.+\left.2 \Lambda_{t}\left|c_{1}\right|\left|c_{2}\right|\left|\psi_{1}\right|\right|_{t}\left|\psi_{2}\right|_{t} \cos \delta_{t}^{\prime}\right],
\end{aligned}
$$

with

$$
\Lambda_{t}=\frac{2\left|\alpha_{t}\right|}{\left(1+\left|\alpha_{t}\right|^{2}\right)} .
$$

For the sake of simplicity, we have assumed that the phase difference between the environment states (included in $\delta_{t}^{\prime}$ ) is constant. Equation (27) is the quantum analog of the optical (phenomenological) equation (16). Thus, $\Lambda_{t}$ is defined as the quantum coherence degree, which plays here the same role as $\mathcal{A}$ in Eq. (16).

Now, we make the (reasonable) assumption that the environment states are too complex to keep mutual coherence as time increases [26]. In this way, even if they are initially coherent, they become orthogonal as time passes. Thus, one can assume $\left|\alpha_{t}\right| \simeq e^{-t / \tau_{c}}, \tau_{c}$ being the coherence time, a measure of how fast the system becomes classical (smaller $\tau_{c}$ implies faster loss of coherence). By substituting the value of $\left|\alpha_{t}\right|$ in Eq. (28) one obtains

$$
\Lambda_{t}=\operatorname{sech}\left(t / \tau_{c}\right),
$$

which establishes a relationship between the coherence degree and coherence time. Although the value of the coherence time can be derived analytically for interfering waves by means of simple Markovian models [9], Eq. (29) allows us to determine it from the coherence degree. In our case, we have used $\Lambda_{t}=0.63$ (or, equivalently, a coherence time of $\tau_{c} \simeq 5.08 \times 10^{-2} \mathrm{~s}$ ), for which an excellent agreement between the experimental data and the theoretical results is found (see below). This value corresponds to a fringe visibility $\mathcal{V}=0.607$ (notice that $\Lambda_{t} \simeq \mathcal{V}$, as said at the end of Sec. III), very close to the experimental value $(\sim 4 \%$ above this value).

Taking all these ingredients into account, we present next the results for two basic models. In the first one, after passing through the corresponding slits, $\left|\Psi_{1}\right|^{2}$ and $\left|\Psi_{2}\right|^{2}$ are considered to be quasiplane waves; while in the second one, their profiles are modeled with Gaussian functions. We also assume that the slits are on the $X Y$ plane, the direction of propagation of the beam is along $Z$, and $\ell_{y} \gg \ell_{x}\left(\ell_{x}\right.$ and $\ell_{y}$ being the dimensions of the slits). In this way, the model can be considered as two dimensional, with the motion taking place in the $X Z$ plane. In order to minimize the effects of the spreading of the wave function along $z$ with time, given by the general expression
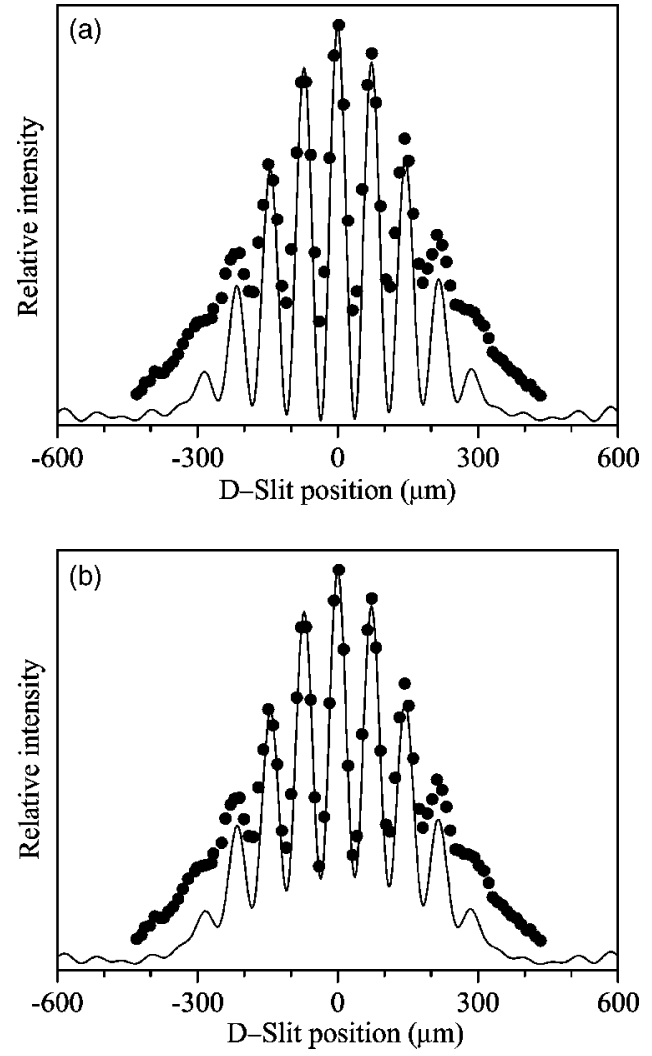

FIG. 2. Intensity obtained from the interference of two quasiplane waves in the case of (a) only incoherence effects, and (b) incoherence plus decoherence effects. For comparison, the experimental data (solid circles) are also shown.

$$
\sigma_{t}=\sigma_{0} \sqrt{1+\left(\frac{\hbar t}{m \sigma_{0}^{2}}\right)^{2}}
$$

it is enough, in our case, to choose $\sigma_{0}^{z}=2 \bar{a}$, which ensures $\sigma_{t} \simeq \sigma_{0}$ during the time of propagation.

\section{A. Case I: Quasiplane waves}

In this first case, the probability density at the left slit has been simulated by covering its width with 30 Gaussian packets, while for the right (wider) one 31 packets have been used. For both slits $z_{0}=0$ for all Gaussians, and the distance between neighbor packets has been taken equal to a constant, $\Delta_{i}=a_{i} /\left(N_{i}-1\right)$, with $i=1,2$. The width parameter along the $X$ direction is $\sigma_{x}^{(i)}=a_{i} / N_{i}$, with $N_{i}=30$ or 31 depending on the slit considered, while for the $Z$ direction $\sigma_{z}=2 \bar{a}$, as explained above. The time evolution of the wave function has been numerically obtained using Heller's method [27], which is exact in our case.

In Fig. 2(a) we show the results corresponding to a case where only incoherence effects are included [Eq. (20)]. As mentioned above, after passing through the slits each partial wave has a certain motion component along the perpendicular direction. The (initial) momentum component along each direction can be estimated using the uncertainty principle, according to which $p_{x} \sim \Delta p_{x} \sim \hbar / \Delta x$. Since $\Delta x \sim a$, the momentum along the parallel direction will be $p_{x}^{(i)} \simeq \pm \hbar / a_{i}$, 
with the plus (minus) sign corresponding to the right (left) slit. Hence, the momentum along the $Z$ direction is $p_{z}^{(i)}$ $=\left[\left(2 \pi \hbar / \lambda_{\mathrm{dB}}\right)^{2}-\left(p_{x}^{(i)}\right)^{2}\right]^{1 / 2}$. As can be seen in the figure, the results indicate that the effects due to incoherence are very small, with the minima of the oscillations almost touching the horizontal axis; they display, however, a certain degree of asymmetry with respect to $x=0$. The problem turns out to be quite similar to the evolution of the same initial wave with both slits of the same width and no perpendicular motion. Finally, notice the oscillations that appear for $x \gtrsim 400 \AA$. They arise from the diffractive effects caused by the borders of the slits, and are not observed in the equivalent solid curve of Fig. 1 because of the double-averaging process (with respect to the finite size of $D$ and the bandwidth) carried out there. As will be seen in next section, these oscillations disappear when a model based on Gaussians is used.

To conclude this section, we show in Fig. 2(b) the intensity resulting when both incoherence and decoherence (with $\left.\Lambda_{t}=0.63\right)$ effects are included [Eq. (27)]. As can be seen, these results have been greatly improved with respect to only considering incoherence, showing an important decrease in the fringe visibility. However, the agreement with the experimental data is still poor. It should be stressed that the results have been obtained by means of a reasonable theory based on first principles, with no averaging as in Sec. III.

\section{B. Case II: Gaussian waves}

In this section we show that the results presented in the previous section can be highly improved by simply considering that the slits are Gaussian [28], i.e., the wave function in Eq. (18) is a linear superposition of two Gaussian wave packets. These Gaussian wave packets are centered at $x_{0}^{(1)}$ $=\left(a_{1}-d\right) / 2$ and $x_{0}^{(2)}=\left(a_{2}+d\right) / 2\left(\right.$ with $\left.z_{0}^{(i)}=0\right)$, respectively, and their corresponding width parameters are taken as $\sigma_{x}^{(i)}$ $=a_{i} / 4$ and $\sigma_{z}=2 a$. The value of $\sigma_{x}^{(i)}$ implies that the intensity at the border of the corresponding slit is $\left|\psi_{i}\left( \pm \sigma_{x}^{(i)} / 2\right)\right|^{2} /\left|\psi_{i}\left(x_{i}\right)\right|^{2}=e^{-2}$ when $\left(x-x_{i}\right)=\sigma_{x}^{(i)} / 2$, i.e., approximately $13.5 \%$ of the total intensity. Thus, only a very small part of the wave will be out of the boundaries defined by the borders of the slits. This assumption is in agreement with the experimental observations, since Zeilinger et al. reported, with regard to the error on the slit widths, that "neutrons penetrating through the boron wire along a chord $0.2 \mu \mathrm{m}$ away from the surface are attenuated by more than a factor 1/e," such that these "neutrons would be refracted far out of the diffraction pattern" [8].

In sharp contrast to the case described in the previous subsection, when incoherence is considered in this model (given, as above, by $p_{x}^{(i)} \simeq \pm \hbar / a_{i}$ and $p_{z}^{(i)}=\left[\left(2 \pi \hbar / \lambda_{\mathrm{dB}}\right)^{2}\right.$ $\left.-\left(p_{x}^{(i)}\right)^{2}\right]^{1 / 2}$, for slits with different sizes), the resulting intensity, shown in Fig. 3(a), looks quite different compared with the results of Fig. 2(a). In this case, it can be observed how the Gaussian envelope due to single-slit diffraction (which modulates the amplitude of the intensity maxima) allows the interference pattern to fit the shape of the experimental data fairly well. Nonetheless, the central minima do not completely agree with the experimental ones yet. This theoretical
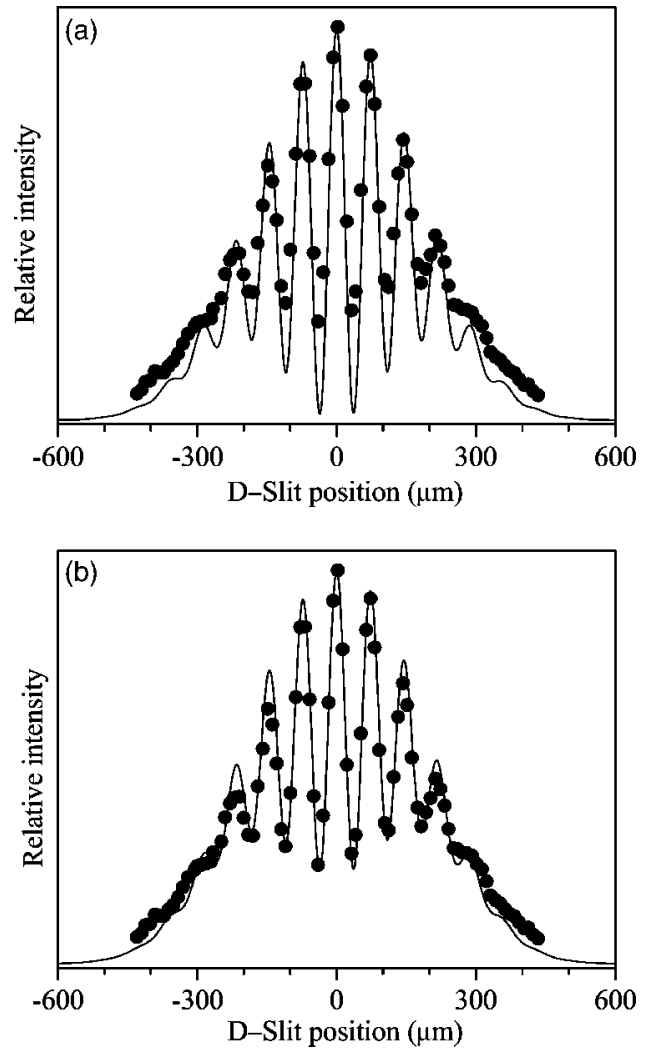

FIG. 3. Intensity obtained from the interference of two Gaussian slits for (a) only incoherence effects, and (b) incoherence plus decoherence effects. For comparison, the experimental data (solid circle) are also shown.

pattern looks very much like that given by Eq. (15) (see results in Fig. 1). Notice that the diffractive effects have disappeared, and then the "tail" of the curve decreases exponentially, as expected. Qualitatively, we can state that this model reproduces the experimental pattern as well as the optical theory, but with the advantage that here we do not need to assume any average.

Finally, when we consider Eq. (27) with $\Lambda_{t}=0.63$, which includes both incoherence and decoherence effects in this Gaussian-slit approach, the agreement between theoretical and experimental results is excellent, as can be seen in Fig. 3(b). Therefore, this experiment cannot be interpreted in terms of only optical models, but needs to include decoherence as a mechanism leading to the loss of coherence. This is not an a priori expected result since the neutron mass is very small.

\section{CONCLUSIONS}

Optical models are currently used in slit diffraction experiments with particles like electrons, neutrons, atoms, or fullerenes in order to explain the behavior of the empirical data. These models are usually based on reasonable physical assumptions about the nature of the particle source, and/or the influence of the different objects placed in the pathway of the particle beam in the experimental setup. Here, we have shown how the two-slit experiment with cold neutrons per- 
formed by Zeilinger et al. [8] can be explained to a certain extent by means of a simple analytical model based on such considerations. This analysis has allowed us to characterize the relevance of the different sources of incoherence present in the experiment.

However, these procedures mask the presence of another relevant phenomenon that is likely to exist, and may have important influence on the experiment: decoherence. This mechanism, also leading to the suppression of coherence in the system, cannot be described by optical models. Decoherence is produced by the dynamical interaction between system and environment (e.g., by means of scattering events), which is not included in such models.

In this paper we have shown that decoherence is likely to exist in Zeilinger et al.'s experiment, and that it can be explained fairly well with a simple model assuming an exponential damping of the interference term [9], and using the experimental value of the coherence degree $\left(\Lambda_{t}=0.63\right)$. By means of this model we have been able to establish the in- fluence of each element of the experimental arrangement on the neutron beam. Among these influences, the most important one is the effect that the double slit causes on the neutron beam: it splits the initial quasiplane wave front coming from $C$ into two coherent Gaussian beams.

\section{ACKNOWLEDGMENTS}

F.B. and M.J.B. gratefully acknowledge Professor T. Seligman for his hospitality at the Cuernavaca CIC (UNAM, Mexico), where this collaboration was started. A.S.S. is deeply indebted to Professor Paul Brumer for interesting discussions on the ideas developed here. This work was supported in part by MCyT (Spain) under Contracts No. BFM2000-347 and No. BQU2003-8212. A.S.S. gratefully acknowledges partial support from the Consejería de Educación y Cultura of the Comunidad Autónoma de Madrid (Spain).
[1] M. Born and E. Wolf, Principles of Optics (Pergamon Press, Oxford, 1980).

[2] L. Mandel and E. Wolf, Rev. Mod. Phys. 37, 231 (1965).

[3] L. E. Ballentine, Quantum Mechanics. A Modern Development (World Scientific Publishing Co., Singapore, 1998).

[4] M. A. Nielsen and I. L. Chuang, Quantum Computation and Quantum Information (Cambridge University Press, Cambridge, U.K., 2000).

[5] D. Giulini, E. Joos, C. Kiefer, J. Kupsch, I.-O. Stamatescu, and H. D. Zeh, Decoherence and the Appearance of a Classical World in Quantum Mechanics (Springer, Berlin, 1996).

[6] E. Joos and H. D. Zeh, Z. Phys. B: Condens. Matter 59, 223 (1985).

[7] C. Kiefer and E. Joos, in Quantum Future, edited by P. Blanchard and A. Jadczyk (Springer, Berlin, 1998).

[8] A. Zeilinger, R. Gähler, W. Treimer, and W. Mampe, Rev. Mod. Phys. 60, 1067 (1988).

[9] C. M. Savage and D. F. Walls, Phys. Rev. A 32, 2316 (1985).

[10] R. E. Grisenti, W. Schöllkopf, J. P. Toennies, J. R. Manson, T. A. Savas, and H. I. Smith, Phys. Rev. A 61, 033608 (2000).

[11] W. Schöllkopf and J. P. Toennies, Science 266, 1345 (1994).

[12] A. Viale, M. Vicari, and N. Zanghì, Phys. Rev. A 68, 063610 (2003); quant-ph/0307160.

[13] H. Rauch and S. A. Werner, Neutron Interferometry (Oxford University Press, New York, 2000).

[14] By "stationary" we mean that the time average of these processes is independent of the choice of the time origin.

[15] E. Wolf, Proc. R. Soc. London, Ser. A 225, 96 (1954); 230, 246 (1955).

[16] A. Papoulis, Systems and Transforms with Applications in Optics (McGraw-Hill, York, 1968).
[17] M. J. Bastiaans, Opt. Acta 24, 261 (1977).

[18] L. Mandel and E. Wolf, J. Opt. Soc. Am. 66, 529 (1976).

[19] In some optical treatments, $C$ and $O$ are considered as input and output planes, respectively. Then the point-spread function $h(x, \xi)$ is interpreted as the response of the system at $x$ (on $O$ ) to a Dirac pulse occurring at $\xi$ (on $C$ ). Accordingly, $\xi$ labels the output coordinate, and $x$ the input one. For more details, see J. W. Goodman, Introduction to Fourier Optics (McGraw-Hill, New York, 1996).

[20] We use the term "correlation pattern" because, as inferred from Eq. (7), the modulation appears in the correlations between two different positions $x_{1}$ and $x_{2}$ on $O$, but not for $x_{1}=x_{2}$. This is a consequence of considering $S$ as an incoherent source. Nonetheless, the pass through $O$ leads to the assumption of a certain coherence for the neutron beam [see Eq. (11), for example].

[21] The inclusion in the calculations of some reasonable degree of noncoherence along the width of the slits does not change the results significantly.

[22] R. Omnès, Rev. Mod. Phys. 64, 339 (1992).

[23] A. O. Caldeira and A. J. Leggett, Physica A 121, 587 (1983).

[24] Time dependence is indicated by a subscript " $t$ " [e.g., $|\Psi(t)\rangle$ $=|\Psi\rangle_{t}$ ], while initial values do not carry any label [e.g., $|\Psi(0)\rangle=|\Psi\rangle]$.

[25] H.-P. Breuer and F. Petruccione, The Theory of Open Quantum Systems (Oxford University Press, Oxford, 2002).

[26] R. Omnès, Phys. Rev. A 56, 3383 (1997).

[27] A. S. Sanz, F. Borondo, and S. Miret-Artés, J. Phys.: Condens. Matter 14, 6109 (2002).

[28] R. P. Feynman and A. R. Hibbs, Quantum Mechanics and Path Integrals (McGraw-Hill, New York, 1965). 